\title{
UC-MAJORIZATION AND ITS STRONGLY LINEAR PRESERVERS
}

\author{
MINA JAMSHIDI
}

Abstract. In this paper we introduce the concept of semimajorization as a generalized form of majorization. After that we discuss uc-majorization. For $x, y \in \mathbb{R}^{n}$, we say $x$ is uc-majorized by $y$ (written as $x \prec_{u c} y$ ) if there exists an upper triangular column stochastic matrix $A$ such that $x=A y$. In our main theorem we characterize all linear maps that strongly preserve $\prec_{u c}$ on $\mathbb{R}^{n}$. Furthermore at the end, we characterize strong linear preservers for lc-majorization.

Mathematics subject classification (2010): 15A04, 15A21.

Keywords and phrases: Semimajorization, uc-majorization, lc-majorization, strong linear preserver linear preserver.

\section{REFERENCES}

[1] T. ANDo, Majorization, Doubly stochastic matrices, and comparision of eigenvalues, Linear Algebra and its Applications 118 (1989), 163-248.

[2] A. Armandnejad And A. Ilkhanizadeh Manesh, GUT-majorization and its linear preservers, Electronic Journal of Linear Algebra 23 (2012) 646-654.

[3] A. ARmandnejad and S. Mohtashami And M. Jamshidi, On linear preservers of g-tridiagonal majorization on $\mathbb{R}^{n}$, Linear Algebra and its Applications 459 (2014) 145-153.

[4] A. Giovagnoli AND H.P. WynN, G-majorization with applications to matrix orderings, Linear Algebra and its Applications 67 (1985) 111-135.

[5] A. M. HASANI AND M. RADJABALIPOUR, The structure of linear operators strongly preserving majorizations of matrices, Electronic Journal of Linear Algebra 15 (2006) 260-268.

[6] F. Khalooei AND A. SAlEmi, The Structure of linear preservers of left matrix majorization on $\mathbb{R}^{p}$, Electronic Journal of Linear Algebra 18 (2009) 88-97.

[7] A. W. Marshall, I. OlKin AND B. C. ARNOLD, Inequalities: Theory of Majorization and Its Applications, Springer, 2011.

[8] M. Soleymani AND A. ARmandneJAD, Linear preservers of circulant majorization on $\mathbb{R}^{n}$, Linear Algebra and its Applications 440 (2014) 286-292.

[9] M. Soleymani AND A. ARmandNeJAD, Linear preserver of even majorization on $M_{n, m}$, Linear Multilinear Algebra 62 (2014) 1437-1449. 